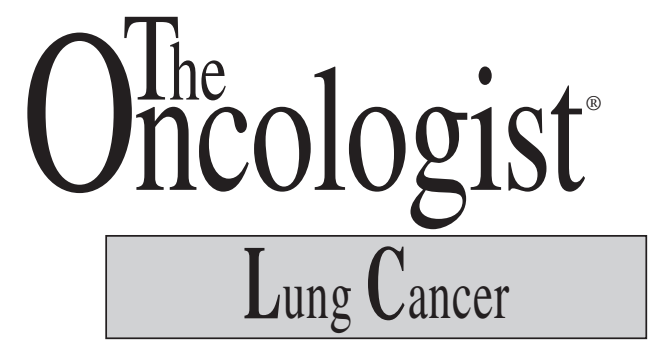

\title{
Outcomes of Research Biopsies in Phase I Clinical Trials: The MD Anderson Cancer Center Experience
}

\author{
Hazem El-Osta, ${ }^{a}$ David Hong, ${ }^{\text {a Jennifer Wheler, }},{ }^{a}$ Siqing Fu, ${ }^{a}$ Aung Naing,,${ }^{\text {a Gerald Falchook, }}{ }^{\text {a }}$ \\ Marshall Hicks, ${ }^{b}$ SiJin Wen, ${ }^{\text {c Apostolia M. Tsimberidou, }}{ }^{a}$ Razelle Kurzrock ${ }^{\text {a }}$ \\ Departments of ${ }^{\mathrm{a} I n v e s t i g a t i o n a l ~ C a n c e r ~ T h e r a p e u t i c s ~(P h a s e ~ I ~ C l i n i c a l ~ T r i a l s ~ P r o g r a m), ~}{ }^{\mathrm{b}}$ Diagnostic Imaging, \\ and ${ }^{\mathrm{c} B i o s t a t i s t i c s, ~ T h e ~ U n i v e r s i t y ~ o f ~ T e x a s ~ M D ~ A n d e r s o n ~ C a n c e r ~ C e n t e r, ~ H o u s t o n, ~ T e x a s, ~ U S A ~}$
}

Key Words. Drug development • Mandatory biopsy • Optional biopsy • Phase I clinical trial • Research biopsy

Disclosures: Hazem El-Osta: None; David Hong: None; Jennifer Wheler: None; Siqing Fu: None; Aung Naing: Research funding/contracted research: National Institutes of Health; Gerald Falchook: None; Marshall Hicks: None; Sijin Wen: None; Apostolia M. Tsimberidou: None; Razelle Kurzrock: None.

The content of this article has been reviewed by independent peer reviewers to ensure that it is balanced, objective, and free from commercial bias. No financial relationships relevant to the content of this article have been disclosed by the independent peer reviewers.

\section{Abstract}

Background. Research biopsies are crucial for exploring the impact of novel agents on putative targets. The current study assesses the safety and success rate associated with performing such biopsies.

Methods. We reviewed the medical records of 155 consecutive patients who had one or more research biopsies as part of a phase I trial from September 2004 to October 2009.

Results. Of 281 research biopsies performed, 118 were paired before and after treatment biopsies (total $=236$ biopsies). The most common sites of biopsy were superficial lymph node (19.9\%), followed by liver (16.4\%), and then soft tissue $(15.7 \%)$. Ultrasound-guided biopsies were the most frequent type (53.7\%). Among 142 patients who consented for mandatory biopsy, 86.6\% had the biopsy performed, compared with $4.4 \%$ of 911 patients offered a biopsy on an optional basis $(p<.0001)$. Biopsy was obtained most frequently on industry-sponsored trials; lack of funding on nonindustry trials was the most common reason that biopsies were not obtained. Of 281 single biopsies, only $4(1.4 \%)$ had complications: pneumothorax requiring chest tube placement $(n=2)$, infection requiring admission $(n=1)$, and arrhythmia with hypotension $(n=$ 1). All but one biopsy was successful in obtaining tissue. No deaths were attributable to biopsy.

Conclusions. Our experience demonstrates that research biopsies in early phase clinical trials are safe (1.4\% risk of serious complications), and a higher percentage of patients underwent mandatory biopsies $(86.6 \%)$ compared with that of the patients with optional biopsies (4.4\%). The Oncologist 2011;16:1292-1298

\section{INTRODUCTION}

Phase I trials play a major role in early drug development, bringing investigational drugs from bench to bedside. Tra- ditionally, phase I trials aim to define the safety, pharmacokinetic profile, and recommended dose of novel drugs for future phase II studies [1]. In addition, these trials describe

Correspondence: David Hong, M.D., Department of Investigational Therapeutics, (Phase 1 Program)-Unit 455, Division of Cancer Medicine, MD Anderson Cancer Center, 1515 Holcombe Boulevard, Houston, Texas 77030, USA. Telephone: 713-563-5844; Pager: 713606-3113; Fax: 713-792-0305; e-mail: dshong@ mdanderson.org Received February 21, 2011; accepted for publication June 16, 2011; first published online in The Oncologist Express on August 22, 2011. @AlphaMed Press 1083-7159/2011/\$30.00/0 http://dx.doi.org/ 10.1634/theoncologist.2011-0043 
response signals, and in the era of targeted therapy, pharmacodynamic properties such as target impact have become increasingly important. Pharmacodynamic studies provide proof-of-principle for the mechanism(s) of action and biologic effect of novel drugs on target molecules. Incorporating these endpoints into early phase I trials is a critical step toward developing targeted agents for patients who are most likely to benefit $[2,3]$.

These new strategies in oncology require collecting samples from tumor or normal tissue before and after treatment. Safety concerns and ethical controversies surrounding research biopsies have been voiced [4]. In particular, there may be concerns regarding the risk of harm without a clearly defined prospect of benefit. In light of the limited published literature on this subject [5], we conducted a retrospective review of the safety of research biopsies performed on patients enrolled in phase I trials at MD Anderson Cancer Center.

\section{Patients ANd Methods}

\section{Data Collection}

We undertook an electronic chart review of 155 consecutive patients enrolled in 45 phase I trials that included a mandatory or optional biopsy for correlative studies in the Department of Investigational Cancer Therapeutics (Phase I Clinical Trials program) at The University of Texas MD Anderson Cancer Center from September 2004 to October 2009. Patient information was analyzed that included age, gender, performance status, body mass index, anticoagulation intake, previous treatments on phase I trials, blood chemistry panels (albumin, platelet, international normalized ratio, activated partial thromboplastin time), and cancer types. We also systematically reviewed data regarding how biopsy tissue was obtained, including site, method, timing, type of needle used, and outcome (failure to obtain tissue, serious complications), and characterized the clinical trials (sponsored by industry or nonindustry trials, mandatory or optional biopsies). Trials that were not sponsored by industry included trials sponsored by the National Cancer Institute, National Comprehensive Cancer Network, or no sponsor. The latter included mainly trials using FDAapproved drugs in combination. We defined serious complications as events that occurred during the first 4 weeks after the biopsy procedure and required immediate surgical or interventional procedures, or hospitalization. We defined comorbidity as any medical condition that coexists with the primary cancer such as hypertension, diabetes mellitus, heart disease, and lung disease. This study and all biopsies were done according to the guidelines of the MD Anderson Cancer Center Institutional Review Board (IRB).

\section{Statistics}

Patient characteristics, biopsy features, and outcomes were summarized using descriptive statistics and exploratory data analysis. Categorical data were described using contingency tables. Continuously scaled measures were summarized with descriptive statistical measures [i.e., mean ( \pm SD) or median (range)]. The $95 \%$ confidence interval of the risk of failure and complications of biopsies was estimated based on binomial distributions. The Fisher's exact test was used to examine the association between two categorical variables. A $p$-value $<.05$ denoted statistical significance. Statistical analysis was performed using SPSS 17.0 software (SPSS, Chicago, IL) and SPLUS (Insightful Corp., Seattle) and was verified by our statistician (S.W.).

\section{RESULTS}

\section{Patient Demographics}

Overall, 45 phase I trials, 13 investigator-initiated, and 32 sponsored studies involving research biopsy on a mandatory or optional basis were identified. A total of 281 biopsies were performed in a total of 155 distinct patients and 163 protocol enrollees. It should be noted that the 163 protocol enrollees biopsied represent 155 distinct patients, because 8 patients who were biopsied enrolled on more than one protocol and were biopsied on both protocols in which they participated. These 155 distinct patients included 110 patients who had at least one paired before and after treatment biopsy ( 8 of whom had two paired biopsies on two different phase I studies). Therefore, there were 118 paired before and after treatment biopsies, or a total of 236 biopsies that were part of a pair. In addition, there were 45 single biopsies of which 42 were before treatment biopsies and 3 were after treatment biopsies. The purpose of these biopsies varied among protocols, and most protocols specified several types of uses, which included immunohistochemistry staining, mutation analysis, single-nucleotide polymorphism analysis, and messenger RNA profiling.

Demographic and laboratory data are shown in Tables 1 and 2. Patient age ranged from 14 to 85 years, with a median of 57 years. Eighty patients were men (51.6\%). Eight patients $(5.2 \%)$ had previous biopsies in the setting of phase I trials, and $46(29.7 \%)$ had been enrolled in earlier phase I trials. Performance status was 0 to 1 in 156 of 163 cases $(95.7 \%)$. Comorbidity at the time of biopsy was confirmed in 95 of 163 cases (58.3\%): hypertension ( $n=71,43.6 \%)$, diabetes mellitus $(n=24,14.7 \%)$, thyroid dysfunction $(n=$ $17,10.4 \%)$, dyslipidemia $(n=18,11 \%)$, heart disease $(n=$ $15,9.2 \%)$, lung and kidney disease $(n=4,2.5 \%$ each), and miscellaneous $(n=15,9.2 \%)$. The main types of tumors were cutaneous (mainly melanoma) $(n=30,19.4 \%)$, gas- 
Table 1. Demographics

\begin{tabular}{|c|c|}
\hline Variable & Results \\
\hline Median age (range) & 57 years $(14-85)$ \\
\hline \multicolumn{2}{|l|}{ Gender $^{\mathrm{a}}$} \\
\hline Male & $80(51.6 \%)$ \\
\hline Female & $75(48.4 \%)$ \\
\hline \multicolumn{2}{|l|}{ Cancer type $^{\mathrm{a}}$} \\
\hline Cutaneous (mainly melanoma) & $30(19.4 \%)$ \\
\hline Gastrointestinal & $30(19.4 \%)$ \\
\hline Head and neck & $17(10.9 \%)$ \\
\hline Thoracic & $16(10.3 \%)$ \\
\hline Gynecologic & $13(8.4 \%)$ \\
\hline Genitourinary & $12(7.7 \%)$ \\
\hline Endocrine (mainly thyroid) & $10(6.5 \%)$ \\
\hline Breast & $10(6.5 \%)$ \\
\hline Soft tissue (mainly sarcoma) & $9(5.8 \%)$ \\
\hline Lymphatic & $8(5.1 \%)$ \\
\hline Previous treatment on phase I trials ${ }^{\mathrm{a}}$ & $46(29.7 \%)$ \\
\hline $\begin{array}{l}\text { Previous biopsy in the setting of } \\
\text { phase I trial }^{\mathrm{a}}\end{array}$ & $8(5.1 \%)$ \\
\hline Anticoagulation ${ }^{\mathrm{b}}$ & $18(11 \%)$ \\
\hline Antiplatelet agents ${ }^{\mathrm{b}}$ & $11(6.7 \%)$ \\
\hline \multirow[t]{4}{*}{ Performance status ${ }^{\mathrm{b}}$} & $0: 55(33.7 \%)$ \\
\hline & $1: 101(62 \%)$ \\
\hline & $2: 6(3.7 \%)$ \\
\hline & $3: 1(0.6 \%)$ \\
\hline \multicolumn{2}{|c|}{$\begin{array}{l}\text { By number of patients (the denominator is the total } \\
\text { number of patients studied, which is } 155 \text { ). } \\
\text { by number of protocol enrollees (the denominator is the } \\
\text { total number of protocol enrollees, which is } 163 \text { because } \\
8 \text { patients who were biopsied were enrolled on more than } \\
\text { one protocol and were biopsied on both protocols in } \\
\text { which they participated). }\end{array}$} \\
\hline
\end{tabular}

trointestinal ( $n=30,19.4 \%)$, head and neck ( $n=17$, $10.9 \%)$, thoracic ( $n=16,10.3 \%)$, gynecologic $(n=13$, $8.4 \%)$, genitourinary $(n=12,7.7 \%)$, endocrine (mainly thyroid) $(n=10,6.5 \%)$, breast $(n=10,6.5 \%)$, soft tissue (mainly sarcoma) $(n=9,5.8 \%)$, and lymphatic $(n=8$, $5.1 \%$ ) (Table 1).

\section{Anticoagulation and Antiplatelet Agents}

Eighteen (11\%) patients were on anticoagulation therapy prior to biopsy; 11 patients $(6.7 \%)$ were on antiplatelet agents. Eight $(4.9 \%)$ of the 18 patients on anticoagulants were on warfarin, whereas $9(5.5 \%)$ were on enoxaparin and $1(0.6 \%)$ was on heparin. Typically, warfarin was held for 5 days before the procedure, whereas enoxaparin was held for 24 hours and aspirin was held for 3 days before the procedure.
Table 2. Biopsy features

\begin{tabular}{|c|c|c|}
\hline & Number & Percentage \\
\hline \multicolumn{3}{|l|}{ Method of biopsy } \\
\hline Ultrasound & 151 & $53.7 \%$ \\
\hline CT scan & 105 & $37.4 \%$ \\
\hline Punch & 23 & $8.2 \%$ \\
\hline Other & 2 (forceps) & $0.7 \%$ \\
\hline Total & 281 & $100 \%$ \\
\hline \multicolumn{3}{|l|}{ Timing of biopsy } \\
\hline Before treatment & 42 & $25.8 \%$ \\
\hline After treatment & 3 & $1.8 \%$ \\
\hline Before and after treatment & 118 & $72.4 \%$ \\
\hline Total & 163 & $100 \%$ \\
\hline \multicolumn{3}{|l|}{ Site of biopsy } \\
\hline Superficial lymph node & 56 & $19.9 \%$ \\
\hline Liver & 46 & $16.4 \%$ \\
\hline Soft tissue & 44 & $15.7 \%$ \\
\hline Lung/pleura & 42 & $14.9 \%$ \\
\hline Abdominal/pelvis & 35 & $12.4 \%$ \\
\hline Skin & 23 & $8.2 \%$ \\
\hline Retroperitoneum & 19 & $6.8 \%$ \\
\hline Kidney/adrenal & 7 & $2.5 \%$ \\
\hline Breast & 5 & $1.8 \%$ \\
\hline Mediastinum & 4 & $1.4 \%$ \\
\hline Total & 281 & $100 \%$ \\
\hline \multicolumn{3}{|l|}{ Needle gauge } \\
\hline 14 & 2 & $0.8 \%$ \\
\hline 16 & 30 & $11.7 \%$ \\
\hline 18 & 175 & $68.3 \%$ \\
\hline 19 & 13 & $5.1 \%$ \\
\hline 20 & 24 & $9.4 \%$ \\
\hline 21 & 2 & $0.8 \%$ \\
\hline 22 & 10 & $3.9 \%$ \\
\hline total & $256^{\mathrm{a}}$ & $100 \%$ \\
\hline \multicolumn{3}{|l|}{ Mandatory versus optional } \\
\hline Mandatory & 123 & $75.5 \%$ \\
\hline Optional & 40 & $24.5 \%$ \\
\hline Total & 163 & $100 \%$ \\
\hline \multicolumn{3}{|l|}{ Industry versus nonindustry } \\
\hline Industry-sponsored & 120 & $73.6 \%$ \\
\hline Nonindustry-sponsored & 43 & $26.4 \%$ \\
\hline Total & 163 & $100 \%$ \\
\hline $\begin{array}{l}\text { aThis number reflect biopsies } \\
\text { does not include punch biopsi } \\
\text { forceps }(N=2) \text {. }\end{array}$ & $\begin{array}{l}\text { tained using } \\
(N=23) \text { or }\end{array}$ & $\begin{array}{l}\text { needle and } \\
\text { oiopsies by }\end{array}$ \\
\hline
\end{tabular}

In only five cases, antiplatelet agents were not held prior to procedure (superficial lymph node biopsy, $n=2$; skin biopsy, $n=1$; breast biopsy, $n=1$; abdominal biopsy, $n=$ 
1). Warfarin was not held before biopsy in one case of superficial lymph node biopsy and one case of a subcutaneous lesion, whereas enoxaparin was not held in one case of a superficial lymph node biopsy. No significant after procedure complications were encountered in the eight patients in whom the anticoagulation or antiplatelet agents were not held prior to the procedure, or in the patients in whom it was held.

\section{Biopsy Characteristics}

The main sites of biopsy included superficial lymph node $(n=56,19.9 \%)$, liver $(n=46,16.4 \%)$, soft tissue $(n=44$, $15.7 \%)$, lung and pleura $(n=42,14.9 \%)$, abdomen and pelvis $(n=35,12.4 \%)$, skin $(n=23,8.2 \%)$, retroperitoneum $(n=19,6.8 \%)$, renal/adrenal $(n=7,2.5 \%)$, breast $(n=5$, $1.8 \%)$, and mediastinum $(n=4,1.4 \%)$. Ultrasound-guided biopsy was the most frequent method for obtaining tissue $(n=151,53.7 \%)$ followed by computed tomographyguided (CT-guided) scan $(n=105,37.4 \%)$, punch skin biopsy $(n=23,8.2 \%)$, and biopsy by forceps $(n=2,0.7 \%)$. Needles of 18 gauge or above were used in most procedures ( $n=224$ of 256 biopsies, $87.5 \%$ ). Most skin punch biopsies were 3-4 $\mathrm{mm}$ in diameter (17 of 23) (Table 2).

Of the patients biopsied, 59.5\% $(n=97)$ were on industry-sponsored trials with mandatory biopsies; $14.1 \%(n=$ 23) were on industry-sponsored trials with optional biopsies; $16 \%(n=26)$ were on nonindustry-sponsored trials with mandatory biopsies; and $10.4 \%(n=17)$ were on nonindustry-sponsored trials with optional biopsies. In regard to "mandatory" biopsies, industry-sponsored trials generally were inflexible about the mandatory requirement. However, even when biopsies were considered "mandatory" in nonindustry-sponsored trials, many protocols allowed enough flexibility so that patients or physicians could opt out of the biopsy providing there was a valid medical or logistical reason. Among 142 protocol enrollees on studies with "mandatory" biopsies, biopsy was done in 123 (86.6\%). Among 911 protocol enrollees offered a biopsy on an optional basis, biopsies were done in only 40 cases $(4.4 \%)$, which was significantly lower $(p<.0001)$. Twenty-six of $45(57.8 \%)$ protocol enrollees considered for mandatory biopsies per protocol on nonindustry-sponsored trials had the biopsy performed compared to 97 of 97 protocol enrollees (100\%) in industry-sponsored trials $(p<$ $.0001)$. The reason for not having the mandatory research biopsy done in the 19 patients was documented as follows: 14 cases due to lack of funding, 2 cases due to medical reason, 2 cases due to patient's refusal of the procedure, and 1 case due to absence of biopsiable lesion. Of the 355 enrollees offered an optional biopsy in industry-sponsored trials, 23 had the biopsy done, compared to only 17 of 556 in non-
Table 3. Comparison of mandatory versus optional biopsies

\begin{tabular}{cllll}
\hline & $\begin{array}{l}\text { Biopsy } \\
\text { Biopsy } \\
\text { done }\end{array}$ & $\begin{array}{l}\text { not } \\
\text { done }\end{array}$ & Total & $\begin{array}{l}\text { \% of } \\
\text { biopsy } \\
\text { done }\end{array}$ \\
\hline $\begin{array}{c}\text { All trials } \\
\text { Mandatory }\end{array}$ & 123 & 19 & 142 & $86.6 \%$ \\
Optional & 40 & 871 & 911 & $4.4 \%$ \\
& 163 & 890 & 1053 & $15.5 \%$
\end{tabular}

Industry-sponsored

trials $^{\mathrm{b}}$

$\begin{array}{lllll}\text { Mandatory } & 97 & 0 & 97 & 100 \% \\ \text { Optional } & 23 & 332 & 355 & 6.5 \% \\ & 120 & 332 & 452 & 26.5 \%\end{array}$

Nonindustry-sponsored

trials ${ }^{c}$

$\begin{array}{lllll}\text { Mandatory } & 26 & 19 & 45 & 57.8 \% \\ \text { Optional } & 17 & 539 & 556 & 3.1 \% \\ & 43 & 558 & 601 & 7.2 \%\end{array}$

Industry versus

nonindustry ${ }^{\mathrm{d}}$

\begin{tabular}{lllll} 
Industry & 120 & 332 & 452 & $26.5 \%$ \\
Nonindustry & 43 & 558 & 601 & $7.2 \%$ \\
& 163 & 890 & 1053 & $15.5 \%$ \\
\hline
\end{tabular}

${ }_{\mathrm{a}}^{\mathrm{a} p<.0001 .}$

${ }^{\mathrm{b}} p<.0001$.

$c_{p}<.0001$
$\mathrm{~d} p .0001$

industry-sponsored trials (6.5\% vs. $3.1 \%, p=.019)$ (Table 3 ). The reasons for not doing the optional biopsies were not specified in the research record or medical chart.

\section{Features of Patients Having Mandatory and Optional Biopsy}

We analyzed and compared the clinical and demographic profiles of patients having optional versus mandatory biopsies. Statistical analyses revealed that women, gynecologic cancer, and breast cancer were more frequently represented in the protocol enrollees who underwent biopsies on studies with optional biopsies compared to those on studies with mandatory biopsies. Indeed, among the 40 protocol enrollees who underwent the biopsy on studies with optional biopsy, 23 were women (57.5\%), 8 had gynecologic cancer (20\%), and 6 had breast cancer (15\%). Otherwise, among the 123 protocol enrollees who underwent the biopsy on studies with mandatory biopsy, 52 were women (42.3\%), 5 had gynecological cancer $(4.1 \%)$, and 4 had breast cancer (3.3\%), with $p$-values of $.10, .003$, and .015 , respectively. The distribution of race between the two biopsy groups was not statistically significant. 


\section{Complications of Research Biopsies}

Of a total of 281 distinct biopsies, only 4 serious complications occurred $(1.4 \%)$. Serious complications included pneumothorax requiring chest tube placement ( $n=2$ of 42 lung biopsies [4.8\%]), skin infection requiring admission $(n=1)$, and supraventricular tachycardia associated with hypotension requiring admission $(n=1)$. No deaths were directly related to biopsy. The two patients having pneumothorax and the one patient who had arrhythmia all recovered promptly. The one patient admitted for skin infection after biopsy died soon thereafter from multiple complications related to cancer, which were not related to the biopsy procedure.

\section{Failure of Research Biopsies}

Research biopsy was unsuccessful in obtaining tissue in only one case. In that patient, an attempt was made to access the hepatic lesion using ultrasound guidance. The needle was advanced into the skin and soft tissues. However, because of poor visualization of the lesion, the procedure was aborted. This patient had a subsequent successful pelvic biopsy.

\section{DISCUSSION}

Assessing the effect of a drug on a specific molecular target in tumor tissue and identifying biomarkers within tumor cells that might correlate with response or resistance has played an increasing role in the discovery of new drugs and in advancing our understanding of cancer biology. Consequently, the use of mandatory or optional research biopsies and the ethics and safety of conducting research biopsies has come to the fore in early clinical trial development. Our study confirms that research biopsies are safe $(1.4 \%$ serious complication rate) similar to studies done in other patient populations. The $1.4 \%$ risk of significant complications that we observed is similar to the risk associated with diagnostic biopsy $[4,6]$ and supports the results of Dowlati et al. [5] in the research setting. Moreover, we have shown here, perhaps for the first time, that mandatory biopsies have a much higher success rate $(86.6 \%)$ of obtaining necessary tissue than optional biopsies (4.4\%), and an even higher success rate on protocols with minimal protocol flexibility concerning mandatory biopsies (100\%).

Ethical criteria related to medical research have been established with the intention of protecting patients from harm and exploitation and ensuring their autonomy and privacy [7]. There has been debate about the requirements for research-related biopsy procedures, especially when "mandatory." Criticism has centered upon potential harm in the absence of direct benefit to patient care [4], impact on patient autonomy, and issues related to unrealistic expecta- tions from patients regarding benefit despite informed consent [8-11]. There are limited data about the risks associated with research biopsies in the early clinical trials setting for patients with advanced cancer. Our experience is that single or sequential research biopsies in early phase clinical trials can be performed safely and successfully when adequate precautions are taken and experienced operators are involved. Only $1.4 \%$ of our 155 patients had a serious complication, and there were no deaths caused by the biopsies. Three of the four serious complications were reversible, and in the fourth case the patient died soon thereafter of progressive cancer. No bleeding was seen, perhaps in part because of the policy of stopping anticoagulation or antiplatelet agents for several days before the procedure. Despite the high incidence of repeat thromboembolism in this population, no associated morbidity emerged. Most of our patients had a good performance status despite the extent of their disease, and this might have contributed to their low biopsy morbidity.

Regarding technique, an 18-gauge needle biopsy was used in most cases, but use of a 14-gauge needle has been reported to be safe and to yield a greater amount of tissue [5, 12]. Of our patients, $53.7 \%$ had an ultrasound-guided biopsy, and this method appeared as safe and effective as CT scan-guided biopsies, but is significantly less expensive at our institution. The highest risk in our study was in the subset of patients who had lung biopsies. There were two cases of pneumothorax requiring chest tubes out of a total of 42 single lung biopsies (4.8\%), a similar result from a previous report in the literature [13]. In another reported multivariate analysis of 660 CT-guided lung biopsies [14], the incidence of pneumothorax requiring chest tube insertion was $1 \%$. This study showed that the risk factors for the highest rate of pneumothorax were lesions $<2 \mathrm{~cm}$ in size, a subpleural lesion depth from the point of pleural puncture to the nearest edge of the lesion of 1-20 mm, and a less experienced operator. Both of our two patients with this complication had a lesion depth between 1 and $20 \mathrm{~mm}$.

Several questions surrounding mandatory biopsies have been raised in the literature. Does requiring a mandatory biopsy better address the scientific question being explored in a trial by increasing the number of collected specimens? Or does such a requirement dissuade patients from enrolling into clinical trials that require mandatory biopsies for research purposes? Given the fact that only about $2 \%-4 \%$ of all adult patients with cancer enroll in clinical trials [15], impact on study participation has not been well explored. In a recent survey-based study, $36 \%$ of oncologists and IRB members believed that requiring a research biopsy might deter patients from participating in clinical trials, and many believed that research biopsies should remain optional [16]. 
However, most oncologists and IRB members perceived that requiring a patient to undergo a mandatory biopsy is ethical [16]. Our data confirm that unless biopsies are mandatory without protocol flexibility they are often not done. Indeed, the rate of biopsies performed in such circumstances was $100 \%$ versus $57.8 \%$ for protocols where biopsies were "mandatory" but with protocol flexibility for medical or logistical reasons. Most of these trials with protocol flexibility were investigator-initiated studies and allowed patients to continue on study for logistical or medical reasons at the discretion of the principal investigator even though the patient was not able to undergo a biopsy. After review of the research records and discussion with the respective principal investigators, the most common reason for not performing the biopsy was financial constraints of the investigator and not the medical condition of the patient or other logistical reasons, that is, in one National Cancer Institute-Cancer Therapy Evaluation Program (NCI-CTEP)sponsored study, translational research funding was ended before all biopsies were performed. The latter data suggest that it may be that adequate finances may be as or more important to procuring a biopsy than the "mandatory" requirement. On the basis of these results, it is not surprising that industry-sponsored trials were most rigid in their demand for biopsies and most successful in their acquisition.

In contrast, only $4.4 \%$ of patients had biopsies performed when they were optional. The research and medical records in our study did not allow us to determine the reasons for patients not undergoing a research biopsy. However, other investigators have also noted the low rate of biopsies that are actually performed when offered on a voluntary basis, with reasons that include: (a) patient-related factors, that is, hesitancy because of weighing the associated risk in the absence of direct benefit and the time commitment [16]; (b) physician-related factors, that is, physicians having a lower acceptability for risk and who are hesitant because of any additional time that might be incurred before obtaining informed consent [17]; and (c) logistical factors, such as lack of funding.

It is interesting to note that the distribution of cancer types seen in this cohort differs from the overall distribution of cancer types seen in the phase I group, as reported in a previous publication from our department [13]. Particularly, there is more cutaneous cancer, mainly melanoma, in our cohort versus those that have been previously reported, which were primarily gastrointestinal malignancies. This might be reflective of the ease and lower cost of skin-punch biopsies in melanoma patients.

One weakness of our study was the lack of data on the tumor yield associated with the biopsies; these data were not available because most samples did not undergo pathology re- view at our institution but instead were reviewed by the sponsor, and the results were not immediately available for this study. However, this does not diminish the significance of our findings on the safety and rate of obtained biopsies.

Although it is well established that the risk and purpose of research biopsies must be effectively communicated to the involved patients [18], whether or not trials should be permitted to mandate biopsies is still a matter of debate, although a consensus appears to have emerged that such a requirement is ethical. It remains unclear if a negative impact on protocol accrual might outweigh the advantages of getting biopsy specimens. Further studies comparing accrual rates between early studies that require mandatory biopsies versus those that do not need to be considered are needed. In addition, further exploration of why patients agree to optional biopsies or decline participation in trials with mandatory biopsies needs to be explored. We are currently conducting a large survey of our phase I patients to determine which factors and characteristics influence a patient's decision to participate in studies with biopsies.

Recent phase I studies, of BRAF inhibitors in BRAF V600E mutated melanoma and anaplastic lymphoma kinase inhibitors in EML-anaplastic lymphoma kinase translocated non-small cell lung cancer patients, have shown the importance of predictive markers obtained from tissue in selecting patients and increasing response rates [19-21]. These types of studies will likely be the model of future phase I studies. The importance of selection by biomarker testing in this model is clear, and biopsies are central to this process [21]. In conclusion, our study shows that biopsies done in a phase I setting are not only safe but should be mandatory and gives further support to these selectionbased early trials.

\section{ACKNOWLEDGMENTS}

This research was supported in part by Grant RR024148 from the National Center for Research Resources, a component of the NIH Roadmap for Medical Research (http://nihroadmap. nih.gov/clinicalresearch/overview-translational.asp). H.E.-O. and D.H. contributed equally to this work. We thank Joann Aaron for editorial assistance.

\section{Author Contributions}

Conception/Design: Hazem El-Osta, David Hong, Jennifer Wheler

Provision of study material or patients: Hazem El-Osta, David Hong, Jennifer Wheler, Siqing Fu, Aung Naing, Gerald Falchook, Marshall Hicks, Sijin Wen, Apostolia M. Tsimberidou, Razelle Kurzrock

Collection and/or assembly of data: Hazem El-Osta, David Hong, Aung Naing, Gerald Falchook, Marshall Hicks, Sijin Wen, Apostolia M. Tsimberidou, Razelle Kurzrock

Data analysis and interpretation: Hazem El-Osta, David Hong, Jennifer Wheler, Siqing Fu, Aung Naing, Gerald Falchook

Manuscript writing: Hazem El-Osta, David Hong

Final approval of manuscript: Hazem El-Osta, David Hong, Jennifer Wheler, Siqing Fu, Aung Naing, Gerald Falchook, Marshall Hicks, Sijin Wen, Apostolia M. Tsimberidou, Razelle Kurzrock 


\section{REFERENCES}

1 Horstmann E, McCabe MS, Grochow L et al. Risks and benefits of phase 1 oncology trials, 1991 through 2002. N Engl J Med 2005;352:895-904.

2 Mandrekar SJ, Sargent DJ. Clinical trial designs for predictive biomarker validation:theoretical considerations and practical challenges. J Clin Oncol 2009;27:4027-4034.

3 Le Tourneau C, Lee JJ, Siu LL. Dose escalation methods in phase I cancer clinical trials. J Natl Cancer Inst 2009;101:708-720.

4 Peppercorn J, Shapira I, Collyar D et al. Ethics of mandatory research biopsy for correlative end points within clinical trials in oncology. J Clin Oncol 2010;28:2635-2640.

5 Dowlati A, Haaga J, Remick SC et al. Sequential tumor biopsies in early phase clinical trials of anticancer agents for pharmacodynamic evaluation. Clin Cancer Res 2001;7:2971-2976.

6 West J, Card TR. Reduced mortality rates following elective, percutaneous liver biopsies. Gastroenterology 2010;139:1230-1237.

7 Stewart DJ, Whitney SN, Kurzrock R. Equipoise lost: ethics, costs, and the regulation of cancer clinical research. J Clin Oncol 2010;28:2925-2935.

8 Helft PR, Daugherty CK. Are we taking without giving in return? The ethics of research-related biopsies and the benefits of clinical trial participation. J Clin Oncol 2006;24:4793-4795.

9 Daugherty C, Ratain MJ, Grochowski E et al. Perceptions of cancer patients and their physicians involved in phase I trials. J Clin Oncol 1995;13:10621072.

10 Nurgat ZA, Craig W, Campbell NC et al. Patient motivations surrounding participation in phase I and phase II clinical trials of cancer chemotherapy. Br J Cancer 2005;92:1001-1005.
11 Kodish E, Stocking C, Ratain MJ et al. Ethical issues in phase I oncology research: a comparison of investigators and institutional review board chairpersons. J Clin Oncol 1992;10:1810-1816.

12 Plecha DM, Goodwin DW, Rowland DY et al. Liver biopsy: effects of biopsy needle caliber on bleeding and tissue recovery. Radiology 1997;204: 101-104.

13 Jain RK, Lee JJ, Hong D et al. Phase I oncology studies: evidence that in the era of targeted therapies patients on lower doses do not fare worse. Clin Cancer Res 2010;16:1289-1297.

14 Yeow KM, Su IH, Pan KT et al. Risk factors of pneumothorax and bleeding:multivariate analysis of $660 \mathrm{CT}$-guided coaxial cutting needle lung biopsies. Chest 2004;126:748-754.

15 Murthy VH, Krumholz HM, Gross CP. Participation in cancer clinical trials: race-, sex-, and age-based disparities. JAMA 2004;291:2720-2726.

16 Agulnik M, Oza AM, Pond GR et al. Impact and perceptions of mandatory tumor biopsies for correlative studies in clinical trials of novel anticancer agents. J Clin Oncol 2006;24:4801-4807.

17 Ranjan S, Chandra P, Prabhu S. Antidepressant-induced bruxism: need for buspirone? Int J Neuropsychopharmacol 2006;9:485-487.

18 Cox AC, Fallowfield LJ, Jenkins VA. Communication and informed consent in phase 1 trials: a review of the literature. Support Care Cancer 2006; 14:303-309.

19 Kwak EL, Bang YJ, Camidge DR et al. Anaplastic lymphoma kinase inhibition in non-small-cell lung cancer. N Engl J Med 2010;363:1693-1703.

20 Flaherty KT, Puzanov I, Kim KB et al. Inhibition of mutated, activated BRAF in metastatic melanoma. N Engl J Med 2010;363:809-819.

21 Chabner BA. New results will change the paradigm for phase I trials and drug approval. The Oncologist 2010;15:1023-1025. 